\title{
Intervenção cirúrgica de um canino incluso em sínfise mandibular: relato de caso
}

\section{Surgical intervention of an unerupted canine in mandibular sinfisis: case report}

\author{
Josfran da Silva Ferreira Filho* \\ Samuel Rocha França** \\ Lana Karine Araújo*** \\ José James de Negreiros Pereira ${ }^{* * *}$ \\ lara Freire Costa Belchior ${ }^{* * *}$ \\ Marcelo Bonifácio da Silva Sampieri
}

\section{Resumo}

Objetivo: relatar um caso de exodontia do dente 43, incluso, na região do mento, com o auxílio de um exame complementar eficaz e de baixo custo: radiografia lateral de mandíbula utilizando filme oclusal. Relato de caso: paciente do gênero masculino, 26 anos de idade, melanoderma e normossistêmico, buscou tratamento ortodôntico por motivos de apinhamento dental anterior e presença do dente 83 (canino inferior decíduo) não esfoliado. Para um planejamento cirúrgico correto, foi realizada a radiografia lateral do mento com filme oclusal, em que se constatou que o dente 43 encontrava-se por vestibular em relação aos dentes anteriores inferiores. Assim, o paciente foi submetido à cirurgia sob anestesia local, para exodontia do dente 43. Considerações finais: o tratamento para transmigração de caninos inferiores deve incluir minuciosos planejamentos clínico e radiográfico. O tratamento sugerido para a grande maioria dos casos é a remoção cirúrgica, devido à possibilidade de falhas dos outros meios de tratamento, ocasionando complicações posteriores.

Palavras-chave: Dente não erupcionado. Mandíbula. Radiografia dental.

\section{Introdução}

Os caninos maxilares e mandibulares, quando estão em sua posição normal, são importantes tanto do ponto de vista estético como do funcional. Porém, caninos permanentes inclusos ocorrem de maneira relativamente comum e estão sendo gradualmente documentados na literatura. ${ }^{1}$ Em alguns estudos, ${ }^{2,3}$ observou-se que os caninos superiores e inferiores inclusos ocorrem em $2 \%$ e $0,2 \%$ da população, respectivamente, sendo a frequência de inclusão entre caninos permanentes 10 vezes maior na maxila do que na mandíbula. Quando o canino inferior se apresenta fora da sua localização habitual, normalmente próximo à linha média, trata-se de uma transmigração. Outro estudo ${ }^{4}$ relata que o canino permanente mandibular é o único dente do arco dental capaz de cruzar a linha média. Sugere-se que os caninos inclusos são mais prevalentes nesse tipo de alteração, devido a condições ambientais, sistêmicas ou locais, possuindo variadas combinações etiológicas..$^{5-7}$ Entre elas, estão: fatores traumáticos, falta de espaço, longo trajeto de erupção do germe dentário do canino, perda prematura da dentição decídua, comprimento anormal da coroa e fatores hereditários. Clinicamente, a maioria dos caninos inclusos encontra-se assintomática, não sendo relatado dor ou desconforto pelo paciente. ${ }^{7}$ Nesses casos, o tratamento consiste em sua remoção cirúr- 
gica. Entretanto, são necessários exames por imagem, como a radiografia panorâmica, a radiografia oclusal ou a tomografia computadorizada por feixe cônico, para auxiliar no diagnóstico correto e no planejamento cirúrgico. ${ }^{8}$ Portanto, o objetivo deste trabalho é relatar um caso de remoção cirúrgica de um canino incluso em região mentual, cruzando a linha média com o auxílio da radiografia lateral de mandíbula utilizando o filme oclusal.

\section{Relato de caso}

Um paciente do gênero masculino, 26 anos de idade, melanoderma, buscou tratamento ortodôntico por motivos de apinhamento dental anterior e presença do dente 83 (canino inferior decíduo) não esfoliado. Foi realizada uma radiografia panorâmica, com finalidade ortodôntica, na qual se diagnosticou a transmigração do dente 43 (canino inferior permanente) na linha média, em região mentoniana, em posição mesioangular (Figura 1A). Ao finalizar o tratamento ortodôntico, o paciente foi atendido no ambulatório do projeto Liga de Anatomia e Traumatologia Buco-Maxilo-Facial (Latium), da Universidade Federal do Ceará, para remoção do dente 43.

Figura 1 - A) Presenca do canino inferior incluso cruzando discretamente a linha média em região mentoniana na radiografia panorâmica; B) Radiografia lateral do mento com filme oclusal.

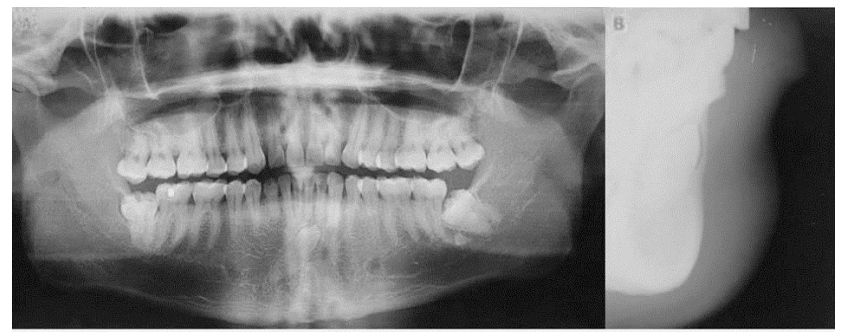

Figura 1: A - Radiografia Panorâmica onde se observa a presença do canino inferior incluso cruzando discretamente a liuha media em região mentoniana, B - Radiografia Lateral do mento com filme oclusal.

Fonte: autores.

Para um planejamento cirúrgico correto, foi realizada a radiografia lateral do mento com filme oclusal (Figura 1B), em que se constatou que o dente 43 encontrava-se por vestibular em relação aos dentes anteriores inferiores. Foi realizada anamnese detalhada do paciente, e não foi detectado nenhum comprometimento sistêmico que contraindicasse o procedimento cirúrgico. Assim, o paciente foi submetido à cirurgia sob anestesia local (lidocaína $2 \%$ e fenilefrina 1:2500), com bloqueio bilateral dos nervos mentuais e anestesia infiltrativa dos nervos incisivos inferiores. Foi realizada uma incisão trapezoidal, para confecção de retalho total do tipo Neumann modificado - gengiva inserida e livre e papila dental com duas incisões relaxantes -, na região do dente 33 ao 83 , expondo a porção óssea anterior da mandíbula (Figura 2C). Em seguida, foram feitas ostectomia e odontossecção para remoção do dente (Figura 2G), com auxílio de brocas $\mathrm{n}^{\circ} 6{\text { e } \mathrm{n}^{\circ}}^{\circ}$ 702 , respectivamente, acopladas à turbina de alta rotação, sob abundante irrigação (Figuras $2 \mathrm{D}$ e $2 \mathrm{E}$ ). Após curetagem, foi realizada sutura da mucosa com fio de algodão 3.0, reposicionando todas as papilas (Figuras 2F), e manteve-se a sutura por 7 dias.
Figura 2 - C) Incisão trapezoidal e divulsão do tecido mole para acesso ao mento; D) Ostectomia; E) Remoção do dente e leito cirúrgico; F) Sutura realizada com fio de algodão 3.0; G) Dente removido.

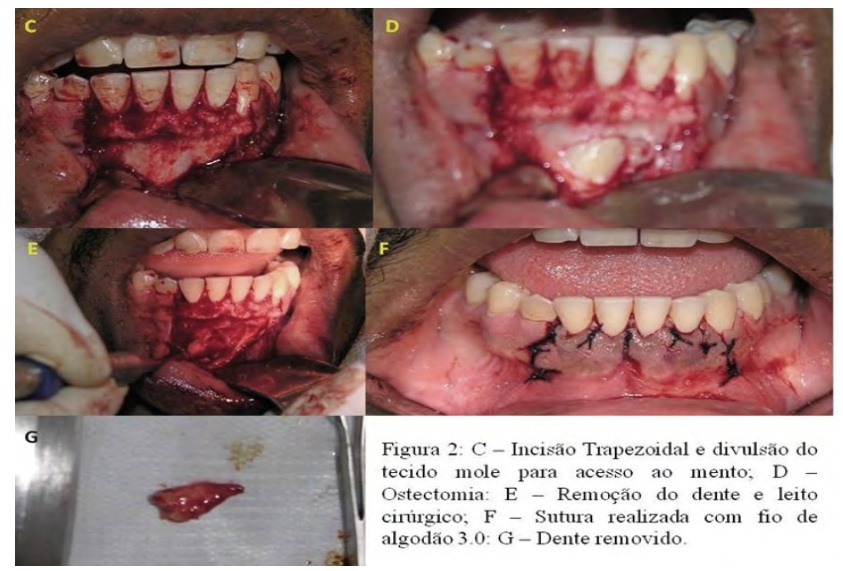

Fonte: autores.

O paciente foi orientado acerca dos cuidados pós-operatórios. A prescrição medicamentosa consistia em antibiótico (Amoxicilina $500 \mathrm{mg}$ de $8 \mathrm{em} 8$ horas durante 7 dias), anti-inflamatório (Ibuprofeno $600 \mathrm{mg}$ de 8 em 8 horas durante 4 dias) e analgésico (Dipirona Sódica $500 \mathrm{mg}$ de 6 em 6 horas durante 3 dias) em caso de dor. Após sete dias, o paciente compareceu para a remoção de sutura, negando queixa álgica ou parestesia. Os tecidos moles da região operada apresentavam-se com boa condição cicatricial e sem sinais de infecção (Figura $3 \mathrm{H}$ ). Foi feito um acompanhamento do paciente, que retornou após dois meses sem queixas, sem alterações locais e com ótima cicatrização da ferida cirúrgica (Figuras 3I e 3J).

Figura 3 - H) Pós-operatório de 7 dias; I) Pós-operatório de 2 meses; J) Radiografia lateral do mento com filme oclusal no pós-operatório de 2 meses.

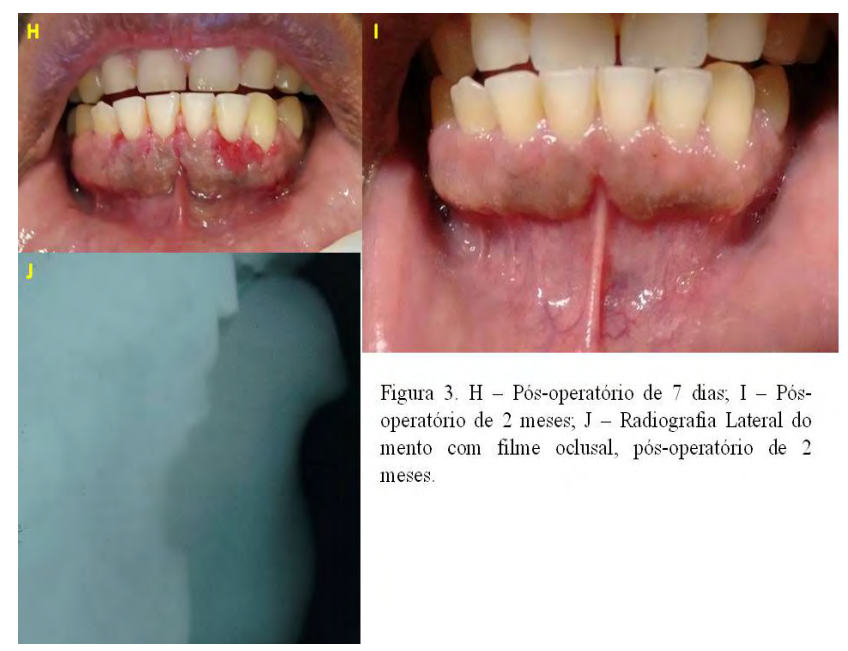

Fonte: autores. 


\section{Discussão}

Em alguns estudos, ${ }^{8-10}$ a frequência de caninos inclusos é mais comum na maxila do que na mandíbula. No entanto, quando ocorre a transmigração de dentes, evento raro, que acomete somente a dentição permanente, são mais comumente caninos mandibulares, ${ }^{8}$ corroborando com os achados no caso em questão. Essa migração dentária cruza a linha média, isto é, a região de sínfise mandibular. No presente trabalho, foi diagnosticada a presença do dente 43 incluso em paciente do gênero masculino, contrapondo-se à literatura, que tem demonstrado que a incidência de caninos transmigrados é maior nas mulheres, e que o lado esquerdo é mais comumente acometido do que o lado direito. ${ }^{1}$ Originalmente, a transmigração foi definida como a condição em que o comprimento total do canino afetado havia migrado para atravessar a linha média da mandíbula. ${ }^{4}$ No entanto, a definição foi modificada, sugerindo que metade ou mais do comprimento do dente precisa atravessar a linha média, para ser considerada transmigração. Embora essa definição encontre suporte, também sugere que não é a distância de migração que é relevante, mas a tendência de caninos para atravessar a linha média, classificando-os em cinco tipos, como mostra o Quadro $1 .^{4}$

Quadro 1 - Classificação dos caninos mandibulares transmigrantes

\begin{tabular}{|l|l|}
\hline Tipo 1 & $\begin{array}{l}\text { O canino incluso está em posição mesioangular, } \\
\text { vestibular ou lingual para os dentes anteriores, com a } \\
\text { coroa cruzando a linha média (45,6\% de todos os casos } \\
\text { publicados). }\end{array}$ \\
\hline Tipo 2 & $\begin{array}{l}\text { O canino é incluso horizontalmente abaixo dos ápices } \\
\text { dos incisivos, perto do limite inferior da mandíbula (20\% } \\
\text { de todos os casos). }\end{array}$ \\
\hline Tipo 3 & $\begin{array}{l}\text { O canino entra em erupção mesial ou distalmente para o } \\
\text { canino oposto (14\% de todos os casos). }\end{array}$ \\
\hline Tipo 5 & $\begin{array}{l}\text { O canino é incluso horizontalmente perto da borda da } \\
\text { mandíbula, abaixo dos ápices de pré-molares ou molares } \\
\text { do lado oposto (17\% de todos os casos). }\end{array}$ \\
$\begin{array}{l}\text { O canino está posicionado verticalmente na linha média, } \\
\text { com o longo do eixo cruzando a linha média (1,5\% de } \\
\text { todos os casos). }\end{array}$ \\
\hline
\end{tabular}

Fonte: adaptado de Mazinis et al. ${ }^{4}(2012)$.

No caso apresentado, o canino encontrava-se na região de sínfise (Tipo 1), com a coroa dentária ultrapassando a região de linha média, embora em outros casos possa atravessá-la e assumir uma posição no lado oposto da mandíbula. ${ }^{4,7}$ Os exames por imagem são muito importantes para o planejamento cirúrgico correto, e a radiografia panorâmica ainda é a mais utilizada. ${ }^{10}$ No caso em questão, utilizaram-se tanto a radiografia panorâmica quanto a lateral de mandíbula empregando filme oclusal. Alguns autores ${ }^{3,5}$ afirmam que os filmes oclusais também ajudam na determinação da posição vestibulolingual do canino incluso e, em conjunto com os filmes periapicais, proporcionam a sua visualização no sentido vestibulolingual. Para auxiliar no diagnóstico e na posição do dente, foi realizada uma radiografia lateral com filme oclusal, o que permitiu a visualização do dente em uma posição vestibular em relação aos incisivos inferiores. Essa técnica apresenta vantagens, como baixo custo e simplicidade da técnica. Outra técnica que pode ser utilizada é a tomografia computadorizada por feixe cônico, que permite uma visualização tridimensional, por meio de cortes e reconstruções multiplanares, fornecendo distância das estruturas adjacentes e condições patológicas associadas. ${ }^{2}$ Apesar de fornecer maior riqueza de detalhes, apresenta maior dose de radiação e maior custo, quando comparada com a técnica utilizada em questão.

No que diz respeito ao tratamento, há consenso dos autores, que citam como formas de tratamento: o tracionamento ortodôntico, o reimplante e a remoção cirúrgica. ${ }^{2,5,8}$ No entanto, no presente trabalho, foi realizado o tratamento cirúrgico, devido à posição do dente, que se apresentava em transmigração $\square$ parte de sua coroa já havia cruzado a linha média $\square$, sendo o procedimento mais empregado. ${ }^{1,2,9}$ Neste caso de inclusão dentária, a remoção cirúrgica é indicada devido à possível associação com lesões patológicas, infecção, lesão aos dentes vizinhos, dor, erupção ectópica e interferência com próteses. ${ }^{1} \mathrm{O}$ acesso intrabucal é a opção para a remoção desses dentes. A remoção de dentes inclusos frequentemente segue os protocolos cirúrgicos básicos de retalho mucoperiosteal e ostectomia já estabelecidos, como no caso em questão. No entanto, existem situações que necessitam de técnicas alternativas para a conclusão do procedimento. A escolha do tratamento depende diretamente de fatores como rizogênese incompleta, idade do paciente e grau de inclusão. ${ }^{10}$ Outra alternativa pode ser o acompanhamento radiográfico do dente incluso sem qualquer intervenção. A desvantagem deste tratamento é que o dente incluso pode gerar complicações ao longo do tempo, havendo a necessidade de uma intervenção cirúrgica tardia e mórbida.

\section{Considerações finais}

$\mathrm{Na}$ maioria dos casos, os dentes inclusos não apresentam qualquer sinal ou sintoma, sendo identificados apenas em exames radiográficos. A radiografia panorâmica é o exame de escolha para diagnosticar caninos e outros dentes inclusos. O tratamento para transmigração de caninos inferiores deve incluir minuciosos planejamentos clínico e radiográfico. $\mathrm{O}$ tratamento sugerido para a grande maioria dos casos é a remoção cirúrgica, devido à possibilidade de falhas dos outros meios de tratamento, ocasionando complicações posteriores. 


\section{Abstract}

Objective: this paper aims to describe and discuss a surgical removal of canine included in the chin region with the aid of an effective and low-cost complementary exam: lateral radiographic of the mandible using the occlusal film. Case report: a male patient, 26 years old, without systemic diseases sought orthodontic treatment due to anterior crowding and presence of an unerupted tooth 83 . In order to perform a correct surgical planning, the lateral radiograph of the occlusal film was performed, where it was found that the tooth 43 was in vestibular position in relation to the lower anterior teeth. Thus, the patient underwent surgery under local anesthesia for tooth extraction. Final considerations: the treatment for transmigration of lower canines should include careful clinical and radiographic planning. The suggested treatment for the vast majority of cases is surgical removal due to the possibility of failure of the other means of treatment, causing subsequent complications.

Keywords: Unerupted tooth. Mandible. Dental radiograph.

\section{Referências}

1. Hyppolito JOP. Tratamento cirúrgico de canino incluso em mento: relato de caso. Rev Odontol UNESP, Araraquara 2011; 40(1):42-6.

2. Dalessandri D, Parrini S, Rubiano R, Gallone D, Migliorati M. Impacted and transmigrant mandibular canines incidence, aetiology, and treatment: a systematic review. Euro J of Orthodontics 2017, 39(2):161-169.

3. Botticelli S, Verna C, Cattaneo PM, Heidmann J J, Melsen B. Two versus three-dimensional imaging in subjects with unerupted maxillary canines. Eur J Orthod 2011; 33(4):344-9.

4. Mazinis E, Zafeiriadis A, Karathanasis A, Lambrianidis T. Transmigration of impacted canines: prevalence, management and implications on tooth structure and pulp vitality of adjacent teeth. Clin Oral Invest 2012; 16:625-32.
5. Sing P, Davies HT. An ectopic tooth concealing an odontogenic myxoma. Dent Update 2013; 40(1):32-5.

6. Rushton MN, Rushton VE. A study to determine the added value of 740 screening panoramic radiographs compared to intraoral radiography in the management of adult ( $>18$ years) dentate patients in a primary care setting. J Dent 2012; 40(8):661-9.

7. Peixoto LR, Gonzaga AK, Melo SL, Pontual ML, Pontual A, De Melo DP. The effect of two enhancement tools on the assessment of the relationship between third molars and the inferior alveolar canal. J Craniomaxillofac Surg 2015; 43(5):637-42.

8. Kruse C, Spin-Neto R, Wenzel A, Kirkevang LL. Cone Beam Computed Tomography and periapical lesions: a systematic review analyzing studies on diagnostic efficacy by a hierarchical model. Int Endod J 2015; 48(9):815-28.

9. Nascimento EHL, Oenning ACC, Freire BB, Gaêta-Araujo H, Haiter-Neto F, Freitas DQ. Comparison of panoramic radiography and cone beam CT in the assessment of juxta-apical radiolucency. Dentomaxillofac Radiol 2018; 47(1):115-23.

10. Sampieri MBS, Correa DS, Viana FLP, Imada TS, Filho JSF, Sant'anna E. Association between imaging and clinical findings in mandibular third molar surgeries. Rev Facul Odont 2018; 23(1):7-11.

\section{Endereço para correspondência:}

Josfran da Silva Ferreira Filho

Rua Dona Leopoldina, 1045, bloco D, ap. 1901, Centro

60110-001, Fortaleza, Ceará, Brasil

Telefone: (88) 99762-3464

E-mail: josfranf@hotmail.com

Recebido: 03/09/18. Aceito: 29/09/18. 